\title{
Successful Treatment of Paecilomyces lilacinus Onychomycosis with Efinaconazole and Tavaborole
}

\author{
John M. Evans ${ }^{a}$ Apphia L. Wang ${ }^{b}$ Boni E. Elewski ${ }^{b}$
}

${ }^{a}$ School of Medicine, and ${ }^{b}$ Department of Dermatology, University of Alabama at Birmingham, Birmingham, Ala., USA

\section{Established Facts}

- Paecilomyces lilacinus is a rare cause of onychomycosis with poor response to conventional antifungals.

\section{Novel Insights}

- We describe successful treatment of onychomycosis caused by $P$. lilacinus with efinaconazole and tavaborole.

- To our knowledge, this is the first case of successfully treated P. lilacinus onychomycosis.

\section{Key Words}

Onychomycosis · Efinaconazole · Tavaborole - Treatment · Non-dermatophyte · Paecilomyces lilacinus · Purpureocillium lilacinum

\begin{abstract}
Paecilomyces lilacinus, also known as Purpureocillium lilacinum, is a non-dermatophyte mold found in the soil and used as nematocide for crops. $P$. lilacinus can cause rare opportunistic infections in humans ranging from endocarditis, keratitis, to onychomycosis with significant resistance to conventional antifungals. There are only two cases of onychomycosis caused by $P$. lilacinus reported in the literature and none that were successfully treated. Here we present a case of successfully treated onychomycosis caused by $P$. lilacinus with
\end{abstract}

(C) 2016 S. Karger AG, Basel

2296-9195/16/0014-0169\$39.50/0 efinaconazole and tavaborole in a patient who had failed treatment with oral fluconazole, itraconazole, terbinafine, and topical ciclopirox and naftifine.

ㄷ) 2016 S. Karger AG, Basel

\section{Introduction}

Paecilomyces lilacinus is a filamentous fungus that can be isolated worldwide from many environmental sources. Recently, there has been an effort to reclassify this organism as Purpureocillium lilacinum, but we will retain the classic name in this discussion [1]. Although uncommon, it can occasionally act as a pathogen especially in the immunocompromised. The most common human infection caused by P. lilacinus is oculomycosis followed by cutane- 


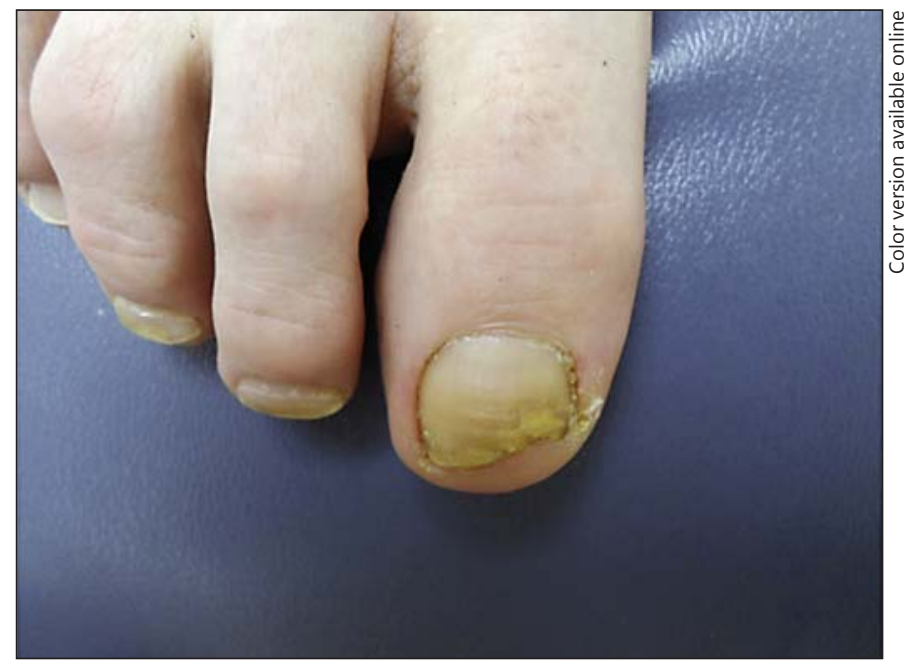

Fig. 1. Right great toenail $P$. lilacinus onychomycosis showing distal onycholysis, subungual debris, and yellow discoloration prior to treatment with topical efinaconazole and tavaborole.

ous infections. Typically, infections caused by this organism are very difficult to treat as it demonstrates resistance to many antifungals [2]. There are only two reports in the literature of onychomycosis caused by $P$. lilacinus, both with limited treatment success $[3,4]$. We herein report a case of a patient with onychomycosis due to P. lilacinus, successfully treated with efinaconazole and tavaborole.

\section{Case Report}

A 68-year-old Caucasian female presented to our clinic for evaluation of a fungal infection of her right great toenail. She first noticed discoloration and dystrophy of this nail at age 13 after having an extensive post-polio corrective surgery performed on her right leg involving bone fusion and relocation of muscle elements. Following this surgery, the patient was fitted with a full right leg cast that extended from the upper right thigh over the entirety of the distal right leg and foot for 3 months. Nail dystrophy on the right great toenail was noted after cast removal and had been continually present since then without involvement of the other toenails.

In 2009, two fungal cultures of her right great toe by the mycology reference laboratory at the University of Alabama showed growth of $P$. lilacinus by colonial and microscopy morphology using modified Sabouraud agar. Oral treatments of fluconazole, itraconazole, and terbinafine only provided temporary mild improvement with return of nail dystrophy and discoloration upon discontinuation of medication; topical ciclopirox and naftifine provided no improvement (fig. 1). Five years after initial culture, daily application of efinaconazole for 4 months revealed improvement of the affected nail. With an additional 2 weeks of intermittent application of tavaborole solution combined with continued daily application of efinaconazole, clearance of onychomycosis was noted accompanied by

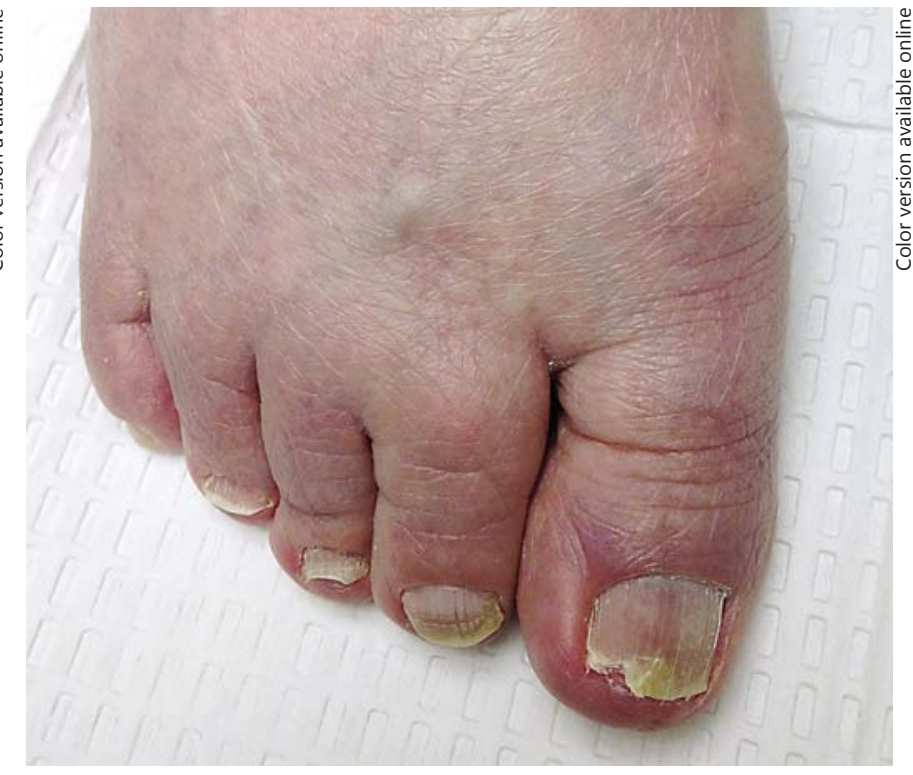

Fig. 2. Right great toenail showing attached distal nail plate without subungual debris or thickened nail bed, status post 4 months treatment with efinaconazole and 2 weeks of tavaborole topical application for P. lilacinus onychomycosis. An incidental nonfungal-related torn nail due to traumatic injury is shown.

minimal onycholysis and an unrelated partial longitudinal separation (fig. 2). The patient was counseled to continue use of tavaborole to minimize risk of recurrence. A potassium hydroxide preparation and fungal culture performed 4 months status post treatment cessation at an accredited laboratory at The University of Alabama at Birmingham and interpreted by a mycologist were negative.

\section{Discussion}

P. lilacinus is classified as a hyaline hyphomycete found globally in the soil and air. Although rarely pathogenic in humans, it most commonly causes cutaneous infections and keratitis in the immunocompromised due to traumatic inoculation [5]. Identification of $P$. lilacinus as a cause of clinical disease has been based on visual identification from two separate cultures, as a single positive culture could result from environmental contamination. Molecular techniques such as ribosomal DNA sequencing can be utilized to increase specificity of organism identification [6].

Unfortunately, $P$. lilacinus is highly resistant to many antifungals such as fluconazole, amphotericin $\mathrm{B}$, and itraconazole. In previous reports of $P$. lilacinus onychomycosis, oral terbinafine and topical amorolfine and clotrimazole have failed to treat the condition $[3,4]$. Interestingly, 
susceptibility has been shown to voriconazole, posaconazole, and terbinafine both in vitro and clinically to $P$. $l i-$ lacinus keratitis and cutaneous infections $[7,8]$.

Onychomycosis is a fungal infection of the nails that has historically been difficult to treat with most cases caused by species from the genera Trichophyton and Epidermophyton [9]. Efinaconazole and tavaborole are topical antifungal drugs approved by the FDA in 2014 demonstrating efficacy in the treatment of onychomycosis caused by dermatophytes in randomized controlled trials. Both drugs have shown in vitro activity against nondermatophyte molds and Candida species, though to our knowledge there have been no published reports of successful treatment of non-dermatophyte onychomycosis with efinaconazole or tavaborole in humans $[10,11]$. We herein report the first case of efinaconazole and tavaborole as clinically effective treatments in non-dermatophyte onychomycosis.

Given that our patient was treated with efinaconazole for nearly 4 months and inconsistently applied tavaborole for 2 weeks, we believe that efinaconazole most likely played a larger role in eradicating our patient's infection, although a synergistic effect cannot be excluded.

\section{Conclusion}

P. lilacinus is a rare cause of onychomycosis with only two previously reported cases, both refractory to treatment. We suggest topical efinaconazole and tavaborole as novel treatment alternatives for $P$. lilacinus-induced onychomycosis.

\section{Statement of Ethics}

Written photographic and informed consent was obtained from the patient described in this article.

\section{Disclosure Statement}

John M. Evans has no conflict of interest to declare. Apphia L. Wang has no conflict of interest to declare. Boni E. Elewski has received honoraria and grants while serving as a consultant and investigator for the following companies: Valeant Pharmaceuticals International Inc.; Anacor Pharmaceuticals, Inc.; Meiji Seika Pharma Co, and Viamet Pharmaceuticals, Inc. This article has no funding source.

\section{References}

1 Luangsa-Ard J, Houbraken J, van Doorn T, Hong SB, Borman AM, Hywel-Jones NL, Samson RA: Purpureocillium, a new genus for the medically important Paecilomyces $l i$ lacinus. FEMS Microbiol Lett 2011;321:141149.

2 Pastor FJ, Guarro J: Clinical manifestations, treatment and outcome of Paecilomyces lilacinus infections. Clin Microbiol Infect 2006;12: 948-960.

3 Fletcher CL, Hay RJ, Midgley G, Moore M: Onychomycosis caused by infection with $\mathrm{Pae}$ cilomyces lilacinus. Br J Dermatol 1998;139: 1133-1135.

4 Innocenti P, Pagani E, Vigl D, Höpfl R, Huemer HP, Larcher C: Persisting Paecilomyces lilacinus nail infection following pregnancy. Mycoses 2011;54:e880-e882.

5 Hall VC, Goyal S, Davis MD, Walsh JS: Cutaneous hyalohyphomycosis caused by Paecilomyces lilacinus: report of three cases and review of the literature. Int J Dermatol 2004;43: 648-653.

6 Iwen PC, Hinrichs SH, Rupp ME: Utilization of the internal transcribed spacer regions as molecular targets to detect and identify human fungal pathogens (review). Med Mycol 2002;40:87-109.

7 Castelli MV, Alastruey-Izquierdo A, Cuesta I, Monzon A, Mellado E, Rodriguez-Tudela JL, Cuenca-Estrella M: Susceptibility testing and molecular classification of Paecilomyces spp.
Antimicrob Agents Chemother 2008;52: 2926-2928.

8 Aguilar C, Pujol I, Sala J, Guarro J: Antifungal susceptibilities of Paecilomyces species. Antimicrob Agents Chemother 1998;42:16011604.

9 Del Rosso JQ: The role of topical antifungal therapy for onychomycosis and the emergence of newer agents. J Clin Aesthet Dermatol 2014;7:10-18

10 Lipner SR, Scher RK: Efinaconazole in the treatment of onychomycosis. Infect Drug Resist 2015;8:163-172.

11 Elewski BE, Tosti A: Tavaborole for the treatment of onychomycosis. Expert Opin Pharmacother 2014;15:1439-1448. 